Introduction and objectives We aimed to study quantitatively the lung cancer pathway from first symptom to treatment and to explore relationship between symptoms, delay and survival.

Methods Newly diagnosed lung cancer patients, referred to Oncology clinics in Norfolk 2008-2012, completed systematic questionnaires regarding date of onset of each symptom, MRC dyspnoea score and $\%$ weight loss. GPs also completed questionnaires. Additional patient, pathway and tumour data were retrieved from hospital records.

The cancer pathway was recorded in five phases: 1) first symptom to GP presentation, 2) to secondary care (SC) referral, 3) to SC appointment, 4) to MDT meeting or date of diagnosis and 5) to treatment commencing.

Results Of 379 patients, mean age was 70.1 years, staging was: III (13.7\%), III (34.3\%) and IV (52\%). Cohort survival was 6.1\% with minimum follow-up of 39 months.

Mean phase lengths were 221.8, 45.8, 10.7, 21.3, 34.7 days for phases 1-5 respectively. Phase 1 was significantly longer $(\mathrm{p}<0.01)$. Mean phase 1 was shorter in stage III-IV than I-II, 200 and 245 days respectively $(\mathrm{p}<0.05)$, in ex-/never-smokers (191.6 days) than smokers $(264.2$ days) $(\mathrm{p}=0.022)$ and if first symptom was haemoptysis compared to cough or dyspnoea. $36.9 \%$ patients felt they delayed seeing their GP. Commonest reasons were: thinking symptoms were insignificant (35\%), anxiety $(28.6 \%)$ and denial (20\%). Good correlation was seen between patient and GP reported dates.

Reported symptoms included (\% initial symptoms in parenthesis): cough 71\% (62.8\%), dyspnoea $62.8 \%$ (27.2\%, chest pain $37.7 \%(8.7 \%)$, haemoptysis 28\% (4.2\%).

Symptoms conferring increased hazard of death were defined as B symptoms. These were: grade 4/5 dyspnoea, hoarseness or loss of voice, metastatic pain and systemic symptoms (HRs 1.77, $1.53,2.21,1.93$ respectively, $\mathrm{p}<0.001)$. Patients with initial cough/mild dyspnoea have means of 127/210 days before B symptoms develop.

Overall no relation was found between phase lengths $1-4$ and survival. Survival increased if phase 5 was $>31$ compared to $\leq 31$ days (HR 0.74, $\mathrm{p}=0.006$ ).

Conclusions Phase 1 is longest. There is no relation between phase length and survival except in phase 5. Symptoms are more important to survival than delay. Effective therapy started within 3 months (before B symptoms) could increase survival.

\section{P81 STRAIGHT TO CT DELIVERS EARLIER FIRST DEFINITIVE TREATMENT IN LUNG CANCER- EFFECT OF A SIMPLE INTERVENTION}

P Malhotra, P Murphy, C Dawson, N Hunt, J Hendry. St Helens and Knowsley Teaching Hospitals NHS Trust, Prescot, UK

\subsection{6/thoraxjnl-2016-209333.224}

Background The National Optimal Lung Cancer Pathway (NOLCP) recommends performing a CT scan before a patients first appointment in a rapid access suspected lung cancer clinic. A local audit in 2014 at our hospital which receives over 350 two week rule suspected lung cancer referrals per year found that less than $50 \%$ of patients had a CT scan before their first appointment.

Objective To determine the effect of a simple cue for physicians stamped on 2 week rule referral forms on the proportion of patients who have a CT scan before their first appointment in a rapid access suspected lung cancer clinic, and its effect on the time to definitive treatment.

Methods This was a retrospective analysis of the lung cancer clinic database at a large district general hospital. Two periods were audited: September - November 2014 (pre-intervention), and July - September 2015 (post-intervention). Data on demographic characteristics, date of first clinic, date of performance of CT scan, and time to definitive treatment was collected. From January 2016 onwards, a simple new intervention was put in place: all 2 week rule referrals were stamped with a cue ("Preclinic CT: Yes or No?") for Consultants triaging the referral to prompt them to arrange a pre-clinic CT scan if appropriate. Reaudit was carried out during the period July-September 2015.

Results Seventy-six out of 81 two week rule referrals between September-November 2014 had a CT scan during their management pathway. Thirty-six (47\%) of these scans were performed before the patients first appointment in clinic. Re-audit between July-September 2015 after introduction of the stamp revealed that $88 \mathrm{CT}$ scans were performed on 101 two week referrals. Of these, $70(80 \%)$ patients had a CT scan before their first appointment.

Time to first definitive treatment improved by 1 week from 38.7 days in the pre-intervention cohort, to 31.5 days in the post-intervention cohort.

Conclusion A simple cue stamped on 2 week rule referral forms increased the proportion of patients who had a CT scan before their first appointment in a rapid access suspected lung cancer clinic from $47 \%$ to $80 \%$, and reduced the time to definitive treatment by 1 week.

\section{P82 OUTCOMES FOR PATIENTS WITH NEGATIVE SCANS ON THE 'STRAIGHT TO CT' PATHWAY}

H Gundersen, A Hufton, R Trafford, MJ Walshaw, M Ledson. Liverpool Heart and Chest Hospital NHS Foundation Trust, Liverpool, UK

\subsection{6/thoraxjnl-2016-209333.225}

Introduction Liverpool has a 'straight to CT' service for patients with coded radiology suspicious of lung cancer and for clinicians worried symptoms may indicate malignancy. In our pathway the lung cancer team automatically take patients whose CT suggests lung cancer. For patients whose scan does not suggest cancer the result is forwarded to their GP to act upon as necessary. We have investigated the outcomes for this patient group for the calendar year 2015 .

Method 387 CT scans were carried out on the 72 hour 'straight to CT' pathway. The cancer services took 179 patients. We have reviewed local radiology, result datasets and hospital records for the remaining 208 patients whose CT results were managed by their GP.

Results Within the group without suggestions of cancer on CT, indications for 72 hour CT were: 90 patients (43\%) had coded radiology and 118 'worried clinician' (57\%) (23\% haemoptysis; $34 \%$ other symptoms). The results of the CT scans showed 42 (20\%) nodules requiring follow up, 49 (23.5\%) inflammatory changes, $49(23.5 \%)$ nil significant, 20 (10\%) emphysema, 11 $(5 \%)$ bronchiectasis and $37(18 \%)$ combinations of other (fibrosis, PE, atelectasis etc). For follow up 17 (8\%) were already under the care of a chest physician, 74 (36\%) were referred to a chest physician and 117 (56\%) were managed in primary care. 73 patients $(35.1 \%)$ had repeat CT scans. In total 99 scans have been done, $31.3 \%$ of these were ordered by primary care $68.7 \%$ by 
secondary care. 4 patients following repeat imaging were diagnosed with cancer. 3 of these had nodules initially, 1 had inflammatory shadowing.

Conclusion The 'straight to CT' pathway dictates that all patients with a CT scan not suggestive of lung cancer remain under the care of the referring clinician. Only $35 \%$ of patients subsequently needed referral for secondary care advice. The 'straight to CT' service not only provides prompt action for patients with cancer but empowers primary care to manage non- malignant diseases. Patients are now managed in the most appropriate setting and inappropriate hospital visits minimised.

\section{P83 INTRODUCTION OF "STRAIGHT TO CT" IN A LUNG CANCER UNIT - TWO YEARS ON}

RM Trafford, A Hufton, M Walshaw, C Smyth, M Ledson. Liverpool Heart and Chest Hospital, Liverpool, UK

\subsection{6/thoraxjnl-2016-209333.226}

Introduction In 2014 we introduced in conjunction with our primary care colleagues a "straight to CT" protocol for patients with suspected lung cancer, to not only to speed up the diagnostic pathway but also to reassure at an early stage patients without the disease. However, some clinicians suggested that this approach may increase the burden of CT scans performed without improving cancer care.

Method "Straight to CT" is available for patients with a CXR coded as concerning for malignancy, or via a general practitioner with concerns based on symptoms and risk factors. Following radiologist review, if appropriate scans are offered within 72 hours: scan positive cases are reviewed by the lung cancer team for onward next investigation, and where the scan is negative the result is faxed by radiology back to the GP. We compared 2015 data with that for 2014, looking for route of referral, investigations performed, and outcome.

Results In 2015 [2014] 464 [468] were eligible for the "straight to CT" pathway. Of these 258 (56\%) [246, 53\%] coded chest Xrays and 206 (44\%) [222, 47\%] 'worried clinician' referrals.

Of the coded CXRs, 24 [22] patients (9\%)[9\%] declined further investigation. Of the 234 [224] who accepted a $72 \mathrm{hr}$ hour CT scan, 149 (64\%) [119, 53\%] had confirmed cancer.

Of the 206 [222] 'worried clinician' referrals, 21 (10\%) [16, $7 \%$ ] patients declined further imaging or assessment, and 32 (16\%) [29, 13\%] were deemed inappropriate. Of the 153 [177] remaining who went on to have 72 hour CT scans only 29 (19\%) [42, 24\%] had cancer confirmed.

Overall, 387 [401] CT scans were carried out. 178 [187] patients were accepted by the cancer services, and 209 [214] patients remained under primary care.

Cancer conversion rates for accepted patients was 70\% [79\%] Conclusions This study has shown that the burden placed on radiological services has remained constant during the two years of our innovative service, and we had previously shown that introducing this protocol did not increase the overall number of scans. We recommend this pathway to other lung cancers units as a way of improving their diagnostic pathway.
P84

THE RELATIONSHIP BETWEEN UNADJUSTED REFERRAL TO TREATMENT TIMES, DISEASE STAGE AND SURVIVAL IN LUNG CANCER

SA Hodgson, KG Blyth. Respiratory Medicine at the Queen Elizabeth University Hospital, Glasgow, UK

\subsection{6/thoraxjnl-2016-209333.227}

Introduction and objectives Cancer waiting times (CWT) targets have helped hospital services evolve to meet the needs of Lung Cancer patients. However, these outcomes are adjusted to allow for perceived clinical complexity or deviation from a 'standard' diagnostic journey. Few patients breach CWT targets in our unit. We performed a retrospective audit to determine the actual time our patients spent on diagnostic pathways and how this related to disease stage and survival.

Methods 377 consecutive patients who presented with Lung Cancer during 2013 were identified from our MDT database. 243/377 (64\%) presented as an inpatient and were excluded. 22/ 134 GP referrals were excluded (insufficient records, aborted investigation (clinical deterioration, patient preference), incomplete staging) leaving 112 cases. Demographics, histology, referral-to-treatment (RTT), referral-to-diagnosis (RTD) and diagnosis-to-treatment (DTT) times were recorded. Overall Survival (OS) based on RTT times and Stage was assessed using Kaplan-Meier methodology.

Results 82/112 (73.2\%) patients had non-small cell lung cancer, $18(16.1 \%)$ had small cell lung cancer and $12(10.7 \%)$ were radiologically-diagnosed. $48 / 112$ patients $(42.9 \%)$ had stage I to IIIA disease. Mean RTD, RTT and DTT times were 43 (SD 55), 69 (SD 45) and 26 (SD 51) days, respectively.
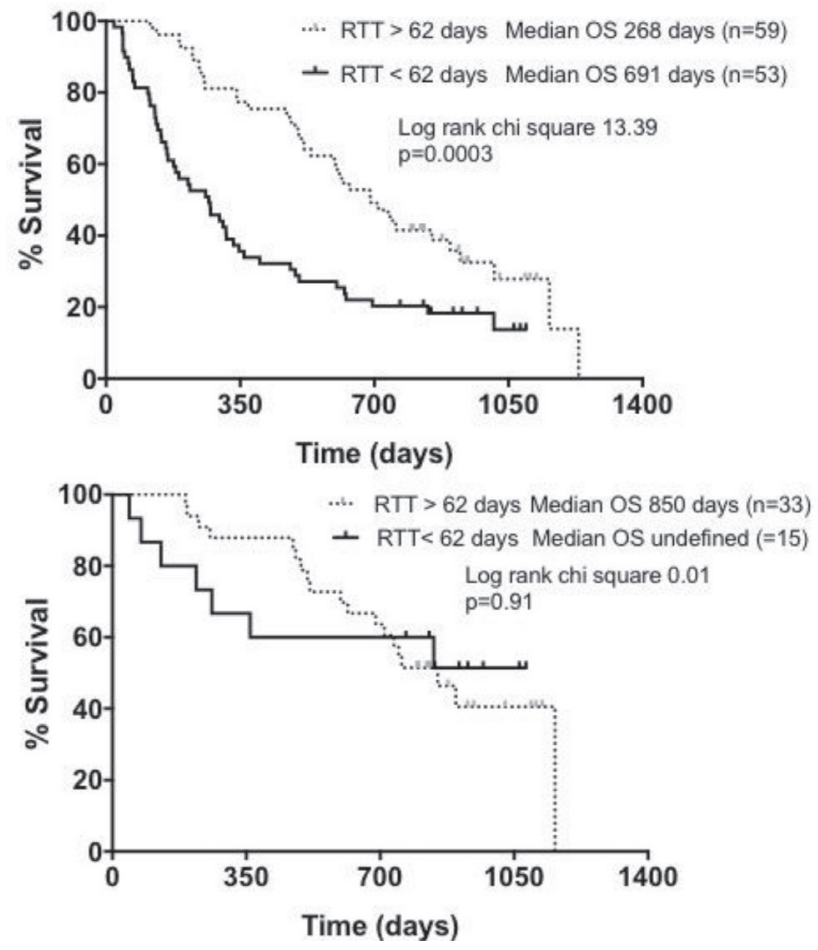

Abstract P84 Figure 1 a) Univariate Survival analysis based on Referral to Treatment time $<$ or $>62$ days in 112 patients with Lung Cancer (all stages) b) Univariate Survival analysis based on Referral to Treatment time $<$ or $>62$ days in 48 patients with potentially radically treatable Stage I-IIIA Lung Cancer 